\title{
Résumé de la Déclaration sur la diarrhée du voyageur du Comité consultatif de la médecine tropicale et de la médecine des voyages (CCMTMV)
}

\author{
Libman $\mathrm{M}^{1}$, au nom du CCMTMV* \\ ${ }^{1}$ Division des maladies infectieuses, Centre universitaire de santé McGill, Montréal (Québec) \\ *Correspondance : CATMAT.Secretariat@phac-aspc.gc.ca
}

\section{Résumé}

Contexte : La plupart des cas de diarrhée du voyageur surviennent lors de voyages dans des pays à revenu faible ou moyen. Le type de voyage, la durée du séjour, l'âge du voyageur et la présence de certains problèmes médicaux sont d'importants facteurs à prendre en compte dans le cas de la diarrhée du voyageur. Le Comité consultatif de la médecine tropicale et de la médecine des voyages (CCMTMV) a formé un groupe de travail sur la diarrhée du voyageur pour mettre à jour les recommandations en matière de diarrhée du voyageur et de voyages internationaux. Le présent document résume la Déclaration concernant la diarrhée du voyageur.

Méthodologie : À la suite d'un examen systématique des études précédentes, des recommandations en matière de prévention et de traitement de la diarrhée du voyageur ont été formulées à l'aide de la méthode Grading of Recommendations, Assessment, Development and Evaluation (GRADE) pour évaluer la qualité des données, les avantages et les inconvénients, ainsi que les valeurs et les préférences des voyageurs. D'autres recommandations ont été fondées sur l'analyse de la documentation et les avis d'experts.

Recommandations : Le CCMTMV s'est servi de la méthode GRADE pour conclure que le vaccin anticholérique oral ne devrait pas être recommandé de manière systématique afin de prévenir la diarrhée du voyageur chez les voyageurs canadiens. Cette recommandation était fondée sur des données de qualité moyenne qui montraient que le vaccin ne permet pas de prévenir la diarrhée du voyageur plus efficacement qu'un placebo. Les options pour la prévention de la diarrhée du voyageur sont le sous-salicylate de bismuth, la fluoroquinolone et la rifaximine (données de grande qualité pour le sous-salicylate de bismuth et les fluoroquinolones, et données probantes modérées pour la rifaximine). En ce qui concerne le traitement de la diarrhée du voyageur, le lopéramide (seul ou avec des antibiotiques), les fluoroquinolones, l'azithromycine et la rifaximine sont des options (données de qualité variable). Le CCMTMV, qui s'appuie sur les données probantes accessibles et l'avis des experts, recommande de se laver les mains ou d'utiliser un désinfectant pour les mains, et de faire preuve de prudence dans le choix et la préparation des boissons et des aliments, ces mesures étant considérées comme des pratiques exemplaires pour prévenir la diarrhée en voyage. De même, aucune recommandation GRADE ne peut pour l'instant être formulée concernant l'utilisation de probiotiques et de prébiotiques pour prévenir la diarrhée du voyageur, ou la prise de sous-salicylate de bismuth pour traiter cette infection, faute de données probantes.

Conclusion : À l'exception du sous-salicylate de bismuth recommandé pour prévenir la diarrhée du voyageur (forte recommandation), toutes les autres recommandations formulées par le CCMTMV à l'égard des agents de prévention et de traitement qui ont été évalués selon la méthode GRADE dans la présente déclaration, sont conditionnelles. Ces recommandations du CCMTMV doivent toujours être prises en considération pour la prévention et le traitement de la diarrhée du voyageur. II faut aussi tenir compte de la situation de chaque voyageur. 


\section{Préambule}

Le Comité consultatif de la médecine tropicale et de la médecine des voyages (CCMTMV) donne de façon continue à l'Agence de la santé publique du Canada (ASPC) des conseils opportuns de nature médicale, scientifique et sanitaire concernant les maladies tropicales infectieuses et les risques pour la santé associés aux voyages internationaux. L'Agence reconnaît que les recommandations et les conseils formulés dans cette déclaration reposent sur les pratiques médicales et les connaissances scientifiques les plus récentes et les diffuse dans le but d'informer les voyageurs ainsi que les professionnels de la santé qui sont appelés à leur prodiguer des soins.

Les personnes qui administrent ou utilisent des médicaments, des vaccins ou d'autres produits devraient bien connaître la monographie des produits, ainsi que toute autre norme ou instruction approuvée concernant leur usage. Les recommandations relatives à l'usage des produits et les autres renseignements présentés ici peuvent différer de ceux qui figurent dans la monographie ou toute autre norme ou instruction approuvée pertinente établie par les fabricants autorisés. Les fabricants font approuver leurs produits et démontrent l'innocuité et l'efficacité de ceux-ci uniquement lorsque ces produits sont utilisés conformément à la monographie ou à toute autre norme ou instruction approuvée semblable.

\section{Introduction}

La diarrhée du voyageur est définie comme étant le passage de trois selles non moulées dans une période de 24 heures, habituellement accompagné d'au moins un symptôme d'une gravité variable, comme de la nausée, des vomissements, des crampes abdominales, de la fièvre et du sang dans les selles (1). Les causes les plus fréquemment associées à la diarrhée du voyageur sont les agents pathogènes bactériens suivants : Escherichia coli (et plus particulièrement les souches entérotoxinogènes et entéroagrégatives) et les campylobactéries (2). Les personnes contractent principalement la diarrhée du voyageur en consommant des aliments ou des boissons contaminés par des agents pathogènes qui causent la diarrhée. La plupart des cas de diarrhée du voyageur surviennent lors de voyages dans des pays à revenu faible ou moyen (3). Le type de voyage, la durée du séjour, l'âge du voyageur et la présence de certains problèmes médicaux sont d'importants facteurs à prendre en compte dans le cas de la diarrhée du voyageur (4).

Les taux d'incidence de la diarrhée du voyageur, chez les personnes qui séjournent dans des régions à haut risque (pays à revenu faible ou moyen) durant une période allant jusqu'à deux semaines, varient de 20 à $90 \%$ (1). La moitié des voyageurs souffrant de diarrhée du voyageur pourraient devoir limiter leurs activités durant leur voyage $(5,6)$, et jusqu'à $10 \%$ des personnes atteintes présenteront une diarrhée persistante, le syndrome du côlon irritable post-infectieux ou d'autres complications (7).

Les options pour la prévention de la diarrhée du voyageur comprennent l'hygiène des mains, les précautions relatives aux aliments et aux boissons, les probiotiques, la vaccination et la chimioprophylaxie. Le traitement de la diarrhée du voyageur consiste en l'utilisation d'agents antisécrétoires, antipéristaltiques et antibiotiques. La réhydratation est un autre aspect important de la gestion de la diarrhée du voyageur, particulièrement chez les enfants.

Le CCMTMV donne de façon continue à l'Agence des conseils opportuns de nature médicale, scientifique et sanitaire concernant les maladies tropicales infectieuses et les risques pour la santé associés aux voyages internationaux. Le présent document résume la Déclaration sur la diarrhée du voyageur du CCMTMV. Une description complète des données probantes et des recommandations est disponible (8).

\section{Méthodologie}

Il s'agit de la deuxième déclaration du CCMTMV pour laquelle on a utilisé la méthodologie GRADE pour formuler des recommandations. GRADE est une méthode pour évaluer la qualité des données probantes et le poids des recommandations dans les lignes directrices qu'utilisent de nombreux organismes internationaux (9). Elle met l'accent sur la transparence et fournit un cadre de travail détaillé qui tient compte des facteurs suivants pour l'élaboration des recommandations : confiance dans l'estimation de l'effet (qualité 
des données), rapport équilibré entre les avantages et les effets néfastes, valeurs et préférences. Les recommandations qui en découlent sont considérées comme « fermes » ou « conditionnelles ». Veuillez vous reporter au Tableau 1 pour connaître les catégories de recommandation GRADE, ainsi qu'à l'appendice ci-dessous pour les questions les plus fréquemment posées à propos de l'interprétation des résultats obtenus au moyen de la méthode GRADE.

Tableau 1: Catégories de recommandations GRADE

\begin{tabular}{|l|l|}
\hline \multicolumn{2}{|c|}{ Catégories de recommandations GRADE ${ }^{1}$} \\
\hline $\begin{array}{l}\text { Ferme }^{2}: \text { Recommandation } \\
\text { FAVORABLE }\end{array}$ & $\begin{array}{l}\text { Le Comité pense que toutes ou presque toutes les personnes } \\
\text { bien informées seraient favorables au plan d'action recommandé } \\
\text { et que seul un petit nombre ne le serait pas. } \\
\text { Conséquences pour les professionnels de la santé : Le } \\
\text { rapport d'équilibre entre les risques et les avantages est tel que } \\
\text { la plupart des voyageurs choisiraient l'intervention. }\end{array}$ \\
\hline $\begin{array}{l}\text { Ferme } \\
\text { DÉFAVORABLE }\end{array}$ & $\begin{array}{l}\text { Le Comité pense que toutes ou presque toutes les personnes } \\
\text { bien informées ne seraient pas favorables au plan d'action } \\
\text { recommandé et que seul un petit nombre le serait. } \\
\text { Implications pour les professionnels de la santé : Le rapport } \\
\text { d'équilibre entre les risques et les avantages est tel que la plupart } \\
\text { des voyageurs ne choisiraient pas l'intervention. }\end{array}$ \\
\hline $\begin{array}{l}\text { Conditionnelle } \\
\text { FAVORABLE }: \text { Recommandation }\end{array}$ & $\begin{array}{l}\text { Le Comité pense que la majorité des personnes bien informées } \\
\text { seraient favorables au plan d'action recommandé, mais qu'une } \\
\text { minorité (peut-être une grande minorité) ne le serait pas. } \\
\text { Conséquences pour les professionnels de la santé : Une } \\
\text { recommandation conditionnelle pourrait inciter les voyageurs à } \\
\text { faire des choix différents. Les professionnels de la santé doivent } \\
\text { présenter aux voyageurs les risques et les avantages de } \\
\text { l'intervention et les aider à prendre une décision qui est } \\
\text { compatible avec leurs valeurs et leurs préférences. }\end{array}$ \\
\hline $\begin{array}{l}\text { Conditionnelle } \\
\text { DÉFAVORABLE }\end{array}$ & $\begin{array}{l}\text { Le Comité pense que la majorité des personnes bien informées } \\
\text { ne seraient pas favorables au plan d'action recommandé, mais } \\
\text { qu'une minorité (peut-être une grande minorité) le serait. } \\
\text { Conséquences pour les professionnels de la santé : Une } \\
\text { recommandation conditionnelle pourrait inciter les voyageurs à } \\
\text { faire des choix différents. Les professionnels de la santé doivent } \\
\text { présenter aux voyageurs les risques et les avantages de } \\
\text { l'intervention et les aider à prendre une décision qui est } \\
\text { compatible avec leurs valeurs et leurs préférences. }\end{array}$ \\
\hline
\end{tabular}

${ }^{1}$ Tiré du manuel et des lignes directrices de la méthode GRADE, sections 14 et 15 (9-11).

${ }^{2}$ Le groupe de travail GRADE semble indiquer qu'il s'attend à ce que $90 \%$ et plus des personnes informées choisissent (ou non) le plan d'action recommandé lorsqu'une recommandation est « ferme ».

${ }^{3}$ Le groupe de travail GRADE semble indiquer qu'il s'attend à ce que moins de $90 \%$ des personnes informées choisissent (ou non) le plan d'action recommandé lorsqu'une recommandation est « conditionnelle ».

\section{Recherches documentaires et résultats}

On a mis en place un cadre d'analyse dans lequel on indique les principales interventions pour la prévention et le traitement de la diarrhée du voyageur. On a dressé une liste de questions clés visant à définir l'ampleur des bienfaits et des effets néfastes et de questions clés concernant la population d'intérêt, les interventions, 
les comparaisons et les résultats. On s'est servi des quatre questions suivantes pour préparer l'évaluation GRADE et formuler les recommandations :

- Chez les voyageurs canadiens, l'administration du vaccin oral inactivé contre le choléra (Dukoral®) réduit-elle le risque de contracter la diarrhée du voyageur par comparaison à la non-vaccination (placebo)?

- Chez les voyageurs canadiens, l'administration d'un agent chimioprophylactique pertinent (c.-à-d. antisécrétoire ou antibiotique) réduit-elle le risque de contracter la diarrhée du voyageur, comparativement à l'absence de chimioprophylaxie (placebo)?

- Chez les Canadiens ayant contracté la diarrhée du voyageur pendant un voyage, l'administration d'un agent thérapeutique pertinent (c.-à-d. antisécrétoire, antipéristaltique ou antibiotique) a-t-elle réduit la durée ou la gravité de la diarrhée du voyageur, comparativement à l'absence de traitement (placebo)?

- Parmi les Canadiens ayant contracté la diarrhée du voyageur pendant un voyage, l'administration d'un agent thérapeutique pertinent (c.-à-d. antisécrétoire, antipéristaltique ou antibiotique) a-t-elle réduit la durée ou la gravité de la diarrhée du voyageur, comparativement à un autre traitement (p. ex. association d'un antipéristaltique et d'un antibiotique; différente classe d'antibiotiques)?

Certaines interventions ne se prêtaient pas à une évaluation GRADE en raison du manque de données probantes valides ou d'interventions de remplacement crédibles qui permettent d'établir des comparaisons. On a aussi élaboré des questions pour formuler les recommandations qui ne sont pas fondées sur la méthode GRADE et qui portent sur les profils de résistance aux antimicrobiens, les précautions relatives à l'eau et aux aliments, l'utilisation d'antibiotiques, de probiotiques, de prébiotiques et de symbiotiques, et la gestion de la déshydratation causée par la diarrhée du voyageur chez les voyageurs.

Plusieurs bases de données électroniques (Ovid MEDLINE, Embase, Global Health et Scopus), de même que la base de données de la revue systématique Cochrane, ont été consultées en employant des variations de l'expression « diarrhée du voyageur », ainsi que le ou les termes de recherche pertinents pour chaque intervention d'intérêt. Les recherches ont porté sur la période allant de la date de création de chaque base de données jusqu'au $1^{\mathrm{er}}$ juin 2013. Pour l'ensemble des recherches, seuls les articles en français ou en anglais ont été retenus. Les listes de références des études pertinentes ont aussi été examinées en vue de recenser des études qui n'auraient pas été relevées durant les recherches dans les bases de données.

Dans notre analyse, la diarrhée du voyageur a été définie comme étant le passage d'au moins trois selles non moulées accompagné d'au moins un symptôme entérique dans une période de 24 heures. On a exclu les études fondées sur une définition moins restrictive de la diarrhée du voyageur pour des questions de cohérence des critères diagnostiques et pour s'assurer que les données probantes qui sont sélectionnées sont axées sur les symptômes importants pour la plupart des voyageurs et des praticiens. Les études visant à évaluer des antibiotiques et le vaccin chez des populations autres que les voyageurs ont été exclues. Cependant, certaines études sur l'utilisation d'antisécrétoires et d'antipéristaltiques chez des populations autres que des voyageurs ont été prises en compte lorsque les données sur les voyageurs étaient limitées; leur inclusion dans l'analyse a toutefois eu pour effet d'abaisser la qualité globale des données.

\section{Évaluation des données probantes}

Tous les détails de la méthodologie GRADE sont fournis ailleurs (12). Essentiellement, la méthode GRADE évalue la qualité des données probantes relatives à des résultats cliniques précis entre les études, et non étude par étude, en corrigeant les défauts liés à la méthodologie, à la cohérence ainsi qu'à la généralisabilité des résultats et de l'efficacité démontrée de l'intervention $(13,14)$. La méthode GRADE tient compte de l'équilibre entre les avantages (efficacité) et les inconvénients des mesures de prévention et des traitements visant la diarrhée du voyageur, du niveau de confiance dans l'estimation de l'effet de chaque intervention (significatif, modéré, faible ou très faible) et de ce que l'on croit être les valeurs et les préférences des voyageurs. Les évaluations de la qualité des résultats d'étude selon la méthode GRADE, ce qui comprend l'efficacité et les effets néfastes associés à chaque intervention, ont été réunies dans un même profil de données probantes et un résumé des tableaux de résultats (8). 
Les recommandations étaient qualifiées de «fermes » ou de " conditionnelles », comme décrites plus tôt (15). D'autres recommandations n'étaient pas fondées sur la méthode GRADE. Elles étaient fondées sur l'évaluation de la documentation pertinente et l'avis des experts.

\section{Résultats}

\section{Prévention de la diarrhée du voyageur}

\section{Vaccin anticholérique oral}

Dukoral $\circledast$ est homologué au Canada pour protéger contre la diarrhée du voyageur et/ou le choléra chez les adultes et les enfants de deux ans et plus qui se rendent dans des régions où il existe un risque élevé de contracter la diarrhée du voyageur due à Escherichia Coli entérotoxinogène ou au choléra causé par $\checkmark$. cholera, et pour prévenir ces affections.

Des données de qualité moyenne montrent que le vaccin anticholérique oral (Dukoral®) (16) n'est pas plus efficace que la vaccination par placebo pour prévenir la diarrhée du voyageur (risque relatif $(R R)=0,94$ et intervalle de confiance (IC) à $95 \%$ : de 0,82 à 1,09) (17-19). Ainsi, $35 \%$ des sujets vaccinés et $37 \%$ des sujets non vaccinés ont souffert de diarrhée. En outre, un examen systématique n'a révélé aucune différence notable entre l'efficacité du vaccin et celle du placebo pour la prévention de la diarrhée du voyageur (20). Aucun effet nocif associé au vaccin et aucune donnée sur les préférences des patients n'ont été recensés.

Certains voyageurs qui présentent un risque élevé de complications ou qui risquent d'être fortement incommodés par la diarrhée du voyageur durant un séjour de courte durée pourraient trouver que les avantages potentiels du vaccin, sur la base de leurs valeurs et préférences personnelles, combinés au faible risque d'effets indésirables, l'emportent sur le fardeau de risque. Pour ces raisons, l'administration du vaccin Dukoral ${ }^{\circledR}$ pourrait toujours être envisagée pour les voyageurs décrits ci-après :

- Les personnes chez qui une brève maladie ne peut être tolérée (c.-à-d. les athlètes de haut niveau, certaines personnes qui voyagent pour affaires ou pour des raisons politiques);

- Les personnes ayant une plus grande sensibilité à la diarrhée du voyageur ( $p$. ex. à cause d'une achlorhydrie, d'une gastrectomie ou d'antécédents de grave diarrhée du voyageur à répétition, les jeunes enfants de plus de 2 ans);

- Les personnes immunodéprimées à cause d'une infection au VIH et dont la numération des lymphocytes T-CD4 est réduite, et les personnes présentant d'autres immunodéficiences;

- Les personnes atteintes de maladies chroniques pour qui le risque de conséquences graves dues à la diarrhée du voyageur est plus élevé (p. ex., les personnes atteintes d'une insuffisance rénale chronique, d'une insuffisance cardiaque congestive, de diabète insulino-dépendant ou d'une maladie inflammatoire chronique de l'intestin).

Il convient de noter que la prise en compte de ces groupes se fonde sur l'opinion d'experts et qu'aucune donnée n'a été publiée sur l'utilisation de Dukoral ${ }^{\circledR}$ dans ces groupes précis.

\section{Sous-salicylate de bismuth}

Des données de grande qualité ont montré que le sous-salicylate de bismuth est plus efficace que le placebo pour prévenir la diarrhée du voyageur (RR =0,55; IC à $95 \%=$ de 0,44 à 0,67) (21-23). De plus, cet effet marqué a été observé quelle que soit la dose administrée (faible ou élevée), et aucune différence n'a été observée entre les deux sous-groupes. Aucun effet nocif grave causé par le sous-salicylate de bismuth et aucune donnée sur les préférences des patients n'ont été recensés.

L'utilisation prolongée du sous-salicylate de bismuth chez les enfants présente un risque d'intoxication par les salicylates et d'encéphalopathie bismuthique, ainsi qu'un risque théorique de syndrome de Reye (24). L'utilisation du sous-salicylate de bismuth peut être autorisée chez certains enfants âgés de deux ans et plus, selon le résultat d'une évaluation individuelle des risques et des avantages. Elle n'est toutefois pas recommandée chez les enfants de moins de deux ans. 


\section{Fluoroquinolones}

Des données de grande qualité ont montré que les fluoroquinolones sont plus efficaces que le placebo pour prévenir la diarrhée du voyageur ( $R R=0,12$; IC à $95 \%=$ de 0,07 à 0,21) (25-28). En outre, un examen systématique a révélé que les fluoroquinolones ont un effet de protection significatif pour la prévention de la diarrhée du voyageur (29). L'administration de fluoroquinolones chez des populations autres que des voyageurs a aussi été associée à de graves effets secondaires, notamment des lésions cartilagineuses, des arthropathies, des ruptures de tendon et des diarrhées dues à $C$. difficile (30-33). La prise de fluoroquinolones chez les voyageurs est également associée à un risque potentiel de sélection d'agents pathogènes résistants aux antimicrobiens (34-39). Une proportion relativement élevée de voyageurs interrogés dans le cadre de l'unique étude descriptive des valeurs et des préférences des voyageurs a indiqué préférer s'abstenir de prendre des antibiotiques afin d'éviter de souffrir de la diarrhée du voyageur (40).

\section{Rifaximine}

Des données de qualité moyenne ont montré que la rifaximine est plus efficace que le placebo pour prévenir la diarrhée du voyageur $(R R=0,42 ;$ IC à $95 \%=$ de 0,33 à 0,53) (41-45). La qualité des données probantes a toutefois été revue à la baisse en raison d'un possible biais de publication, du fait que les résultats d'une vaste étude inscrite dans la base de données des essais cliniques du gouvernement américain n'étaient pas accessibles. Récemment, deux examens systématiques ont révélé que la rifaximine a un effet de protection significatif pour la prévention de la diarrhée du voyageur $(29,46)$. Aucun effet nocif n'a été associé à la prise de rifaximine. Bien qu'aucune association n'ait été établie entre la prise de rifaximine par les voyageurs et la résistance aux antimicrobiens, les risques potentiels devront être surveillés.

\section{Traitement de la diarrhée du voyageur}

\section{Lopéramide}

On a découvert que le lopéramide permettait de réduire la durée et l'intensité de la diarrhée du voyageur chez les voyageurs plus efficacement qu'un placebo ( $p$. ex., risque relatif pour le soulagement initial d'une diarrhée aiguë après quatre heures de traitement $=1,69$; IC à $95 \%=$ de 1,17 à 2,45$)(47-52)$. L'estimation de l'effet a été revue à la baisse en raison du caractère indirect des données probantes, car des études portant sur des populations de non-voyageurs ont été utilisées. Le niveau de confiance dans l'estimation de l'effet a aussi été abaissé pour trois des quatre résultats, à cause d'un nombre insuffisant de sujets (c.-à-d. un manque de précision). Aucun effet nocif n'a été associé à la prise de lopéramide.

Une petite étude indique une augmentation des effets indésirables associés à l'utilisation du diphénoxylate (lomotil, un agent associé au lopéramide) dans le traitement d'une infection à Shigella (53). Le lomotil a un profil d'effet secondaire moins favorable et n'a pas été étudié dans le cadre du traitement de la diarrhée du voyageur.

L'utilisation du lopéramide chez les enfants qui voyagent n'a pas été étudiée. Cependant, un essai comparatif randomisé mené auprès d'enfants âgés de deux à onze ans et présentant une diarrhée aiguë a révélé que le lopéramide avait considérablement réduit la durée et la gravité de la diarrhée, aucune différence n'étant observée entre le groupe traité par le lopéramide et le groupe placebo quant aux effets indésirables associés au médcament (54). Les doses diffèrent selon le groupe d'âge, et le traitement ne doit pas dépasser deux jours. Le lopéramide ne devrait pas être administré aux enfants de moins de deux ans (24). Une forte proportion de voyageurs nord-américains interrogés ont indiqué leur préférence pour un traitement basé sur la prise d'antidiarrhéiques, dont le lopéramide (40).

\section{Lopéramide en association avec des antibiotiques}

L'ajout du lopéramide à l'antibiothérapie a permis de réduire la durée de la diarrhée du voyageur chez les voyageurs comparativement à l'utilisation d'antibiotiques seulement (p. ex., risque relatif pour le soulagement complet de la diarrhée du voyageur après 24 heures $=1,55$; IC à $95 \%=$ de 1,28 à 1,86) $(48$, $55,56)$. Les estimations de l'effet pour deux des quatre résultats ont toutefois été revues à la baisse, en raison d'un écart important entre les études quant à l'orientation de l'effet observé (incohérence). Aucun effet nocif n'a été lié à la prise de lopéramide en association avec des antibiotiques. Une forte proportion de 
voyageurs nord-américains interrogés ont indiqué leur préférence pour un traitement basé sur la prise d'antidiarrhéiques, y compris le lopéramide et des antibiotiques. Étant donné la nature relativement bénigne de la plupart des épisodes de diarrhée du voyageur, et de l'efficacité acceptable des antibiotiques ou du lopéramide utilisés seuls, il est raisonnable de réserver l'association de ces deux médicaments au traitement de la diarrhée grave ou aux patients chez qui le traitement par des antipéristaltiques ou des antibiotiques seuls est inefficace.

\section{Fluoroquinolones}

Des données de qualité moyenne montrent que, comparativement au placebo, les fluoroquinolones sont efficaces pour réduire la durée de la diarrhée du voyageur (risque relatif pour la guérison après 72 heures de traitement $=1,81$; IC à $95 \%$ : de 1,39 à 2,37) $(57,58)$. L'estimation de l'effet a toutefois été revue à la baisse à cause d'imprécisions. L'administration de fluoroquinolones chez des populations autres que des voyageurs a également été associées à des effets indésirables graves et au risque de sélection d'agents pathogènes résistants aux agents antimicrobiens. Ainsi, les fluoroquinolones ne devraient pas être administrées aux enfants de moins de 18 ans pour le traitement de la diarrhée du voyageur, à moins que les avantages de ce traitement ne l'emportent sur les risques potentiels et que les autres options ne puissent s'appliquer.

\section{Azithromycine}

Des données comparant directement l'azithromycine aux fluoroquinolones (et plus particulièrement la ciprofloxacine et la lévofloxacine) montrent que, pour quatre des résultats qui nous intéressent, l'azithromycine est aussi, voire plus efficace que les fluoroquinolones pour réduire la durée de la diarrhée du voyageur ( $p$. ex., risque relatif pour une guérison après 48 heures de traitement $=1,34$; IC à $95 \%=$ de 1,08 à 1,66) (59-62). En ce qui concerne la guérison rapide ou immédiate de la diarrhée du voyageur, les fluoroquinolones se sont révélées plus efficaces que l'azithromycine $(R R=0,46$; IC à $95 \%$ : de 0,25 à 0,84) $(59,61)$. Ces résultats portent à croire que l'azithromycine est aussi efficace que les fluoroquinolones pour soulager la diarrhée du voyageur. On a évalué que la qualité des données était mauvaise. On a revu ces dernières à la baisse à cause de plusieurs facteurs, dont les suivants : nombre insuffisant d'événements pour certains résultats (imprécision); variabilité des résultats entre chaque étude (incohérence) et différences entre les études quant aux doses et à l'utilisation du lopéramide comme traitement d'appoint (caractère indirect). Les données probantes ne semblent pas indiquer d'effets nocifs graves associés à la prise d'azithromycine, bien que des données de faible qualité tirées de deux études indiquent une augmentation du risque de nausées immédiatement après le traitement à l'azithromycine $(R R=6,23 ;$ ICC à $95 \%=$ de 1,48 à 26,26$)(61,62)$. Aucune différence dans les autres mesures des nausées et des vomissements n'a été observée entre ces deux traitements.

\section{Rifaximine}

Des données de grande qualité montrent que la rifaximine est associée à un pourcentage plus élevé de guérison de la diarrhée du voyageur que le placebo $(R R=1,29$; IC à $95 \%=$ de 1,15 à 1,45) $(63,64)$. Des données de grande qualité comparant directement la rifaximine aux fluoroquinolones (ciprofloxacine) n'ont révélé aucune différence significative entre ces deux traitements quant au taux de guérison de la diarrhée du voyageur $(64,65)$. Aucun effet nocif n'a été associé à la prise de rifaximine. Bien qu'aucune association n'ait été établie entre la prise de rifaximine par les voyageurs et la résistance aux antimicrobiens, les risques potentiels devront être surveillés.

\section{Recommandations non fondées sur la méthode GRADE}

On a formulé des recommandations concernant l'hygiène des mains et les précautions relatives aux aliments et à l'eau sans recourir à la méthode GRADE, puisqu'il s'agissait d'interventions préventives, non invasives et douces, et qu'il n'existait aucune intervention de remplacement crédible qui permette d'établir des comparaisons. Le CCMTMV, qui s'appuie sur les données probantes accessibles et l'avis des experts, recommande de se laver les mains ou d'utiliser un désinfectant pour les mains, et de faire preuve de prudence dans le choix et la préparation des boissons et des aliments, ces mesures étant considérées comme des pratiques exemplaires pour prévenir la diarrhée en voyage. De même, aucune recommandation GRADE ne peut pour l'instant être formulée concernant l'utilisation de probiotiques et de prébiotiques pour prévenir la diarrhée du voyageur, ou la prise de sous-salicylate de bismuth pour traiter 
cette infection, faute de données probantes. Vous trouverez un exposé plus approfondi de la documentation accessible sur ces sujets dans la déclaration complète concernant la diarrhée du voyageur (8).

\section{Recommandations et conclusion}

À l'exception du sous-salicylate de bismuth recommandé pour prévenir la diarrhée du voyageur (recommandation « ferme »), toutes les recommandations formulées par le CCMTMV à l'égard des agents de prévention et de traitement qui ont été évalués selon la méthode GRADE dans la présente déclaration, sont conditionnelles (consulter le Tableau 2). Le caractère conditionnel de ces recommandations tient à différents facteurs, dont les faibles effets démontrés, la faiblesse des données à l'appui d'une intervention précise et l'incertitude quant au poids qu'il faudrait donner aux effets nocifs potentiels de l'intervention.

Un des effets nocifs potentiels vient de l'utilisation d'antibiotiques qui pourraient causer une sélection faisant en sorte que l'hôte soit porteur d'agents pathogènes résistants. Des antibiotiques inefficaces pourraient alors être administrés à un voyageur malade pour traiter une diarrhée du voyageur (ou une autre infection). Bien que ce risque ait bien documenté dans d'autres domaines, il n'y a pas de données fiables sur la présence et l'ampleur de ce risque dans le cas de la diarrhée du voyageur. Le CCMTMV recommande que l'on mène des recherches et exerce une surveillance plus systématique en ce qui concerne les profils de résistance des agents pathogènes chez les personnes qui rentrent de voyage et qui ont pris des antibiotiques pour prévenir ou traiter la diarrhée du voyageur. Ces renseignements permettront d'améliorer l'évaluation du risque de base de résistance, en fonction de la destination ou du type de voyage.

Bien que CCMTMV ait une confiance modérée dans les données disponibles lui permettant de formuler une recommandation conditionnelle contre l'administration systématique du vaccin oral contre le choléra (Dukoral $\circledast$ ) pour prévenir la diarrhée du voyageur, d'autres études évaluant l'efficacité de ce vaccin pour prévenir la diarrhée du voyageur seraient nécessaires pour formuler une recommandation plus définitive, pour ou contre l'utilisation du vaccin dans des populations précises.

Tableau 2 : Recommandations fondées sur la méthode GRADE pour la prévention et le traitement de la diarrhée du voyageur chez les voyageurs canadiens

\section{Recommandations fondées sur la méthode GRADE}

Prévention de la diarrhée du voyageur

Recommandations du CCMTMV
- Le vaccin anticholérique oral (Dukoral®) ne devrait pas être administré systématiquement aux voyageurs canadiens.

Recommandation conditionnelle, confiance modérée dans l'estimation de l'effet comparativement au placebo.

Le sous-salicylate de bismuth devrait être envisagé pour les adultes qui présentent un risque important et qui sont disposés à prendre plusieurs doses chaque jour (de 2,1 g à 4,2 g par jour, divisé en quatre doses quotidiennes). Recommandation ferme, confiance élevée dans l'estimation de l'effet comparativement au placebo.

- Un dosage réduit (1,05 g par jour) de sous-salicylate de bismuth pourrait être utilisé dans les situations où un dosage plus élevé est impossible.

Recommandation conditionnelle, faible niveau de confiance dans l'estimation de l'effet comparativement au placebo, faible niveau de confiance quant à l'absence de différence entre les doses faibles et fortes.

- Les fluoroquinolones devraient être considérées comme une option pour prévenir la diarrhée du voyageur chez certaines populations de voyageurs à haut risque qui partent pour une courte période, et pour qui la chimioprophylaxie est considérée comme essentielle. 


\begin{tabular}{|c|c|}
\hline & $\begin{array}{l}\text { Recommandation conditionnelle, confiance élevée dans l'estimation de } \\
\text { l'effet comparativement au placebo. Équilibre entre les avantages et les } \\
\text { inconvénients basé sur les données probantes disponibles sur les effets } \\
\text { indésirables et sur les profils de résistance aux antimicrobiens. } \\
\text { - La rifaximine devrait être considérée comme une option. } \\
\text { Recommandation conditionnelle, confiance modérée dans l'estimation de } \\
\text { l'effet comparativement au placebo. Équilibre entre les avantages et les } \\
\text { inconvénients basé sur les données probantes disponibles sur les profils de } \\
\text { résistance aux antimicrobiens. }\end{array}$ \\
\hline \multicolumn{2}{|r|}{ Traitement de la diarrhée du voyageur } \\
\hline $\begin{array}{l}\text { Recommandations } \\
\text { du CCMTMV }\end{array}$ & $\begin{array}{l}\text { - Le lopéramide devrait être considéré comme une option. } \\
\text { Recommandation conditionnelle, confiance faible à modérée dans } \\
\text { l'estimation de l'effet comparativement au placebo. } \\
\text { - Les fluoroquinolones devraient être considérées comme une option. } \\
\text { Recommandation conditionnelle, confiance modérée dans l'estimation de } \\
\text { l'effet comparativement au placebo. Équilibre entre les avantages et les } \\
\text { inconvénients basé sur les données probantes disponibles sur les effets } \\
\text { indésirables et sur les profils de résistance aux antimicrobiens. } \\
\text { - L'utilisation conjointe du lopéramide et de l'antibiothérapie devrait être considérée } \\
\text { comme une option. } \\
\text { Recommandation conditionnelle, confiance modérée à élevée dans } \\
\text { l'estimation de l'effet comparativement à un antibiotique utilisé seul. } \\
\text { - L'azithromycine devrait être considérée comme une option. } \\
\text { Recommandation conditionnelle, confiance faible dans l'estimation de l'effet } \\
\text { comparativement aux fluoroquinolones. Équilibre entre les avantages et les } \\
\text { inconvénients basé sur les données probantes disponibles sur les profils de } \\
\text { résistance aux antimicrobiens et sur les événements indésirables. } \\
\text { - La rifaximine devrait être considérée comme une option. } \\
\text { Recommandation conditionnelle, confiance élevée dans l'estimation de } \\
\text { l'effet comparativement au placebo, confiance modérée à élevée dans } \\
\text { l'estimation de l'effet comparativement à la ciprofloxacine. Équilibre entre } \\
\text { les avantages et les inconvénients basé sur les données probantes } \\
\text { disponibles sur les profils de résistance aux antimicrobiens. }\end{array}$ \\
\hline \multicolumn{2}{|c|}{ Pratiques exemplaires recommandées pour la prévention de la diarrhée du voyageur } \\
\hline $\begin{array}{l}\text { Recommandations } \\
\text { du CCMTMV }\end{array}$ & $\begin{array}{l}\text { - Se laver les mains avec de l'eau et du savon avant de préparer les repas et de } \\
\text { manger, et après la miction ou la défécation. } \\
\text { - Les désinfectants pour les mains à base d'alcool peuvent contribuer à réduire les } \\
\text { risques de maladies diarrhéiques chez les voyageurs. } \\
\text { - On recommande de ne pas consommer de viande et de fruits de mer } \\
\text { insuffisamment cuits ou crus (66, 67), et d'œufs non pasteurisés (66). Il est } \\
\text { également recommandé d'éviter les aliments qui ont été cuits plus tôt durant la } \\
\text { journée et qui ne sont pas suffisamment réchauffés (68). } \\
\text { - On recommande de ne pas consommer de fruits et légumes qui sont difficiles à } \\
\text { laver (p. ex., les laitues) ou à peler (69), ainsi que les aliments qui sont préparés, } \\
\text { conservés ou servis dans des conditions insalubres (70). } \\
\text { - On recommande d'éviter les aliments humides servis à la température ambiante } \\
\text { (71). Les aliments secs, comme le pain, sont plus sûrs (72). } \\
\text { - Il est relativement sûr de consommer des boissons gazéifiées et alcoolisées en } \\
\text { bouteille en voyage. } \\
\text { - On peut généralement présumer que l'eau plate embouteillée peut être } \\
\text { consommée sans danger, si le scellé du contenant est intact. } \\
\text { - La manière la plus efficace de rendre l'eau potable consiste à la faire bouillir. } \\
\text { La filtration de l'eau doit être suivie d'une désinfection chimique (73). }\end{array}$ \\
\hline
\end{tabular}




\section{Remerciements}

Le présent résumé a été préparé par le groupe de travail sur la diarrhée du voyageur composé de M. Libman (président), de Y. Bui, de J. Geduld, de P. McDonald, de F. Reyes-Domingo et de C. Steensma.

Le CCMTMV tient à remercier Mona Abdel-Motagally pour sa participation à la préparation du résumé, ainsi que le $D^{r}$ Holger Schünemann, professeur titulaire de chaire au département d'épidémiologie clinique et de biostatistiques de l'Université McMaster, pour son soutien en ce qui concerne la méthode GRADE.

Membres du CCMTMV: A. McCarthy (présidente), A. Boggild, J. Brophy, Y. Bui, M. Crockett, W. Ghesquiere, C. Greenaway, A. Henteleff, M. Libman et P. Teitelbaum

Membres de liaison : C. Hui (Société canadienne de pédiatrie) et G. Brunette (Centers for Disease Control and Prevention [États-Unis])

Membres d'office: P. McDonald (Division des médicaments anti-infectieux, Santé Canada), M. Tepper (Direction de la protection de la santé de la Force, ministère de la Défense nationale), P. Charlebois (Centre des services de santé des Forces canadiennes, ministère de la Défense nationale) et S. Schofield (Entomologie de la lutte antiparasitaire, ministère de la Défense nationale)

Membre émérite : C.W.L. Jeanes (décédé en juillet 2014)

\section{Conflit d'intérêts}

Aucun

\section{Financement}

Ce travail a été appuyé par l'Agence de la santé publique du Canada.

\section{Références}

(1) Steffen R. Epidemiology of traveler's diarrhea. Clin Infect Dis. 2005 12/01;41 Suppl 8:S536-40.

(2) Shah N, DuPont HL, Ramsey DJ. Global etiology of travelers' diarrhea: Systematic review from 1973 to the present. Am J Trop Med Hyg. 2009 Apr;80(4):609-14.

(3) Greenwood Z, Black J, Weld L, O'Brien D, Leder K, Von SF, et al. Gastrointestinal infection among international travelers globally. J Travel Med. 2008 07;15(4):221-8.

(4) Kollaritsch H, Paulke-Korinek M, Wiedermann U. Traveler's diarrhea. Infect Dis Clin North Am. 2012 09;26(3):691-706.

(5) Steffen R, Tornieporth N, Clemens SA, Chatterjee S, Cavalcanti AM, Collard F, et al. Epidemiology of travelers' diarrhea: Details of a global survey. J Travel Med. 2004 Jul-Aug;11(4):231-7.

(6) Steffen R, Collard F, Tornieporth N, Campbell-Forrester S, Ashley D, Thompson S, et al. Epidemiology, etiology, and impact of traveler's diarrhea in Jamaica. JAMA. 1999 Mar 3;281(9):811-7.

(7) DuPont HL. Systematic review: The epidemiology and clinical features of travellers' diarrhoea. Aliment Pharmacol Ther. 2009 Aug;30(3):187-96.

(8) Committee to Advise on Tropical Medicine and Travel (CATMAT). Statement on Travellers' Diarrhea. An Advisory Committee Statement (ACS). Ottawa, ON: Public Health Agency of Canada; 2015 Apr. (Disponible en français : www.phac-aspc.gc.ca/tmp-pmv/catmat-ccmtmv/diarrhea-diarrhee-fra.php)

(9) Schunemann HJ, Brozek J, Oxman AD, editors. GRADE handbook for grading quality of evidence and strength of recommendations. Version 3.2 [updated 2009 Mar]

(10) Andrews JC, Guyatt G, Oxman AD, Alderson P, Dahm P, Falck-Ytter Y, et al. GRADE guidelines: 14. Going from evidence to recommendations: The significance and pr0065xsentation of recommendations. J Clin Epidemiol. 2013 Jul;66(7):719-25.

(11) Andrews JC, Schunemann HJ, Oxman AD, Pottie K, Meerpohl JJ, Coello PA, et al. GRADE guidelines: 15. Going from evidence to recommendation-determinants of a recommendation's direction and strength. J Clin Epidemiol. 2013 Jul;66(7):726-35.

(12) Balshem H, Helfand M, Schünemann HJ, Oxman AD, Kunz R, Brozek J, et al. GRADE guidelines: 3 . Rating the quality of evidence. J Clin Epidemiol. 2011;64(4):401-6.

(13) Guyatt GH, Oxman AD, Sultan S, Glasziou P, Akl EA, Alonso-Coello P, et al. GRADE guidelines: 9. Rating up the quality of evidence. J Clin Epidemiol. 2011 Dec;64(12):1311-6. 
(14) Guyatt GH, Oxman AD, Santesso N, Helfand M, Vist G, Kunz R, et al. GRADE guidelines: 12. Preparing summary of findings tables_-binary outcomes. J Clin Epidemiol. 2013 Feb;66(2):158-72.

(15) Committee to Advise on Tropical Medicine and Travel (CATMAT). Statement on International Travellers and Typhoid. Ottawa, ON: Public Health Agency of Canada; 2014. (Disponible en français : http://www.phac-aspc.gc.ca/tmp-pmv/catmat-ccmtmv/friends-amis-fra.php)

(16) Crucell Sweden AB. Dukoral $®$ Product Monograph. 2012.

(17) Peltola H, Siitonen A, Kyronseppa H, Simula I, Mattila L, Oksanen P, et al. Prevention of travellers' diarrhoea by oral B-subunit/whole-cell cholera vaccine. Lancet. 1991;338(8778):1285-9.

(18) Scerpella EG, Sanchez JL, Mathewson JJ, Torres-Cordero JV, Sadoff JC, Svennerholm A, et al. Safety, immunogenicity, and protective efficacy of the whole-cell/recombinant B subunit (WC/rBS) oral cholera vaccine against travelers' diarrhea. J Travel Med. 1995;2(1):22-7.

(19) Wiedermann G, Kollaritsch H, Kundi M, Svennerholm A-, Bjare U. Double-blind, randomized, placebo controlled pilot study evaluating efficacy and reactogenicity of an oral ETEC B-subunit-inactivated whole cell vaccine against travelers' diarrhea (preliminary report). J Travel Med. 2000;7(1):27-9.

(20) Ahmed T, Bhuiyan TR, Zaman K, Qadri F. Vaccines for preventing enterotoxigenic Escherichia coli (ETEC) diarrhoea. The Cochrane Library. 2011.

(21) DuPont HL, Sullivan P, Evans DG. Prevention of traveler's diarrhea (emporiatric enteritis). Prophylactic administration of subsalicylate bismuth. J Am Med Assoc. 1980 1980;243(3):237-41.

(22) DuPont HL, Ericsson CD, Johnson PC, Bitsura JA, de la Cabada FJ. Prevention of travelers' diarrhea by the tablet formulation of bismuth subsalicylate. J Am Med Assoc. 1987;257(10):1347-50.

(23) Steffen R, DuPont HL, Heusser R. Prevention of traveler's diarrhea by the tablet form of Bismuth subsalicylate. Antimicrob Agents Chemother. 1986;29(4):625-7.

(24) Plourde PJ. Travellers' diarrhea in children. Paediatrics \& Child Health. 2003;8(2):99.

(25) Johnson P, Ericsson C, Morgan D, DuPont H, Cabada F. Lack of emergence of resistant fecal flora during successful prophylaxis of traveler's diarrhea with norfloxacin. Antimicrob Agents Chemother. 1986;30(5):671-4.

(26) Heck JE, Staneck JL, Cohen MB, Weckbach LS, Giannella RA, Hawkins J, et al. Prevention of travelers' diarrhea: Ciprofloxacin versus trimethoprim/sulfamethoxazole in adult volunteers working in Latin America and the Caribbean. J Travel Med. 1994;1(3):136-42.

(27) Scott DA, Haberberger RL, Thornton SA, Hyams KC. Norfloxacin for the prophylaxis of travelers' diarrhea in US military personnel. Am J Trop Med Hyg. 1990;42(2):160-4.

(28) Wiström J, Norrby SR, Burman LG, Lundholm R, Jellheden B, Englund G. Norfloxacin versus placebo for prophylaxis against travellers' diarrhoea. J Antimicrob Chemother. 1987;20(4):563-74.

(29) Alajbegovic S, Sanders JW, Atherly DE, Riddle MS. Effectiveness of rifaximin and fluoroquinolones in preventing travelers' diarrhea (TD): A systematic review and meta-analysis. Systematic Reviews. 2009;1(1).

(30) Mackell S. Traveler's diarrhea in the pediatric population: Etiology and impact. Clin Infect Dis. 2005 Dec 1;41 Suppl 8:S547-52.

(31) Noel GJ, Bradley JS, Kauffman RE, Duffy CM, Gerbino PG, Arguedas A, et al. Comparative safety profile of levofloxacin in 2523 children with a focus on four specific musculoskeletal disorders. Pediatr Infect Dis J. 2007 Oct;26(10):879-91.

(32) Neuberger A, Saadi T, Shetern A, Schwartz E. Clostridium difficile infection in travelers-a neglected pathogen? J Travel Med. 2013;20(1):37-43.

(33) Pépin J, Saheb N, Coulombe M, Alary M, Corriveau M, Authier S, et al. Emergence of fluoroquinolones as the predominant risk factor for Clostridium difficile-associated diarrhea: A cohort study during an epidemic in Quebec. Clinical Infectious Diseases. 2005;41(9):1254-60.

(34) Gomi H, Jiang ZD, Adachi JA, Ashley D, Lowe B, Verenkar MP, et al. In vitro antimicrobial susceptibility testing of bacterial enteropathogens causing traveler's diarrhea in four geographic regions. Antimicrob Agents Chemother. 2001;45(1):212-6.

(35) Hoge CW, Gambel JM, Srijan A, Pitarangsi C, Echeverria P. Trends in antibiotic resistance among diarrheal pathogens isolated in Thailand over 15 years. Clinical Infectious Diseases. 1998;26(2):341-5.

(36) Pandey P, Bodhidatta L, Lewis M, Murphy H, Shlim DR, Cave W, et al. Travelers' diarrhea in Nepal: An update on the pathogens and antibiotic resistance. J Travel Med. 2011;18(2):102-8.

(37) Ruiz J, Mensa L, O'Callaghan C, Pons MJ, González A, Vila J, et al. In vitro antimicrobial activity of rifaximin against enteropathogens causing traveler's diarrhea. Diagn Microbiol Infect Dis. 2007;59(4):473-5.

(38) Vila J, Vargas M, Ruiz J, Corachan M, de Anta MTJ, Gascon J. Quinolone resistance in enterotoxigenic Escherichia coli causing diarrhea in travelers to India in comparison with other geographical areas. Antimicrob Agents Chemother. 2000;44(6):1731-3.

(39) Vila J, Vargas M, Ruiz J, Espasa M, Pujol M, Corachan M, et al. Susceptibility patterns of enteroaggregative Escherichia coli associated with traveller's diarrhoea: Emergence of quinolone resistance. J Med Microbiol. 2001;50(11):996-1000.

(40) Ericsson CD, Melgarejo NA, Jelinek T, Mccarthy A. Travelers' preferences for the treatment and prevention of acute diarrhea. J Travel Med. 2009;16(3):172-8. 
(41) Armstrong AW, Ulukan S, Weiner M, Mostafa M, Shaheen H, Nakhla I, et al. A randomized, double-blind, placebo-controlled study evaluating the efficacy and safety of rifaximin for the prevention of travelers' diarrhea in US military personnel deployed to Incirlik Air Base, Incirlik, Turkey. J Travel Med. 2010;17(6):392-4.

(42) Flores J, Dupont HL, Jiang ZD, Okhuysen PC, Melendez-Romero JH, Gonzalez-Estrada A, et al. A randomized, double-blind, pilot study of rifaximin $550 \mathrm{mg}$ versus placebo in the prevention of travelers' diarrhea in Mexico during the dry season. J Travel Med. 2011;18(5):333-6.

(43) DuPont HL, Jiang ZD, Okhuysen PC, Ericsson CD, De La Cabada FJ, Ke S, et al. A randomized, double-blind, placebo-controlled trial of rifaximin to prevent travelers' diarrhea. Ann Intern Med. 2005;142(10):805-12.

(44) Martinez-Sandoval F, Ericsson CD, Jiang ZD, Okhuysen PC, Meléndez Romero JHM, Hernandez N, et al. Prevention of travelers' diarrhea with rifaximin in US travelers to Mexico. J Travel Med. 2010;17(2):111-7.

(45) Zanger P, Nurjadi D, Gabor J, Gaile M, Kremsner PG. Effectiveness of rifaximin in prevention of diarrhoea in individuals travelling to south and southeast Asia: A randomised, double-blind, placebo-controlled, phase 3 trial. The Lancet Infectious Diseases. 2013 Nov;13(11):946-54.

(46) Hu Y, Ren J, Zhan M, Li W, Dai H. Efficacy of rifaximin in prevention of travelers' diarrhea: A meta-analysis of randomized, double-blind, placebo-controlled trials. J Travel Med. 2012;19(6):352-6.

(47) Bergström T, Alestig K, Thoren K, Trollfors B. Symptomatic treatment of acute infectious diarrhoea: Loperamide versus placebo in a double-blind trial. J Infect. 1986;12(1):35-8.

(48) Ericsson CD, DuPont HL, Mathewson JJ, West MS, Johnson PC, Bitsura JAM. Treatment of traveler's diarrhea with sulfamethoxazole and trimethoprim and loperamide. JAMA. 1990;263(2):257-61.

(49) Hughes IW. First-line treatment in acute non-dysenteric diarrhoea: Clinical comparison of loperamide oxide, loperamide and placebo. UK Janssen Research Group of General Practitioners. Br J Clin Pract. 1995 Jul-Aug;49(4):181-5.

(50) Silberschmidt G, Schick MT, Steffen R, Kilpatrick ME, Murphy JR, Oyofo BA, et al. Treatment of travellers' diarrhoea: Zaldaride compared with loperamide and placebo. Eur J Gastroenterol Hepatol. 1995;7(9):871-5.

(51) Van Den Eynden B, Spaepen W. New approaches to the treatment of patients with acute, nonspecific diarrhea: A comparison of the effects of loperamide and loperamide oxide. Current Therapeutic Research. 1995;56(11):1132-41.

(52) Van Loon F, Bennish M, Speelman P, Butler C. Double blind trial of loperamide for treating acute watery diarrhoea in expatriates in Bangladesh. Gut. 1989;30(4):492-5.

(53) DuPont HL, Hornick RB. Adverse effect of lomotil therapy in shigellosis. J Am Med Assoc. 1973;226(13):1525-8.

(54) Kaplan MA, Prior MJ, McKonly KI, DuPont HL, Temple AR, Nelson EB. A multicenter randomized controlled trial of a liquid loperamide product versus placebo in the treatment of acute diarrhea in children. Clin Pediatr. 1999;38(10):579-91.

(55) Dupont HL, Jiang ZD, Belkind-Gerson J, Okhuysen PC, Ericsson CD, Ke S, et al. Treatment of travelers' diarrhea: Randomized trial comparing rifaximin, rifaximin plus loperamide, and loperamide alone. Clinical Gastroenterology and Hepatology. 2007;5(4):451-6.

(56) Ericsson CD, DuPont HL, Okhuysen PC, Jiang ZD, DuPont MW. Loperamide plus azithromycin more effectively treats travelers' diarrhea in Mexico than azithromycin alone. J Travel Med. 2007;14(5):312-9.

(57) Mattila L, Peltola H, Siitonen A, Kyrönseppä H, Simula I, Kataja M. Short-term treatment of traveler's diarrhea with norfloxacin: A double-blind, placebo-controlled study during two seasons. Clinical Infectious Diseases. 1993;17(4):779-82.

(58) Wiström J, Jertborn M, Hedström S, Alestig K, Englund G, Jellheden B, et al. Short-term self-treatment of travellers' diarrhoea with norfloxacin: A placebo-controlled study. J Antimicrob Chemother. 1989;23(6):905-13.

(59) Adachi JA, Ericsson CD, Jiang ZD, DuPont MW, Martinez-Sandoval F, Knirsch C, et al. Azithromycin found to be comparable to levofloxacin for the treatment of US travelers with acute diarrhea acquired in Mexico. Clinical Infectious Diseases. 2003;37(9):1165-71.

(60) Kuschner RA, Trofa AF, Thomas RJ, Hoge CW, Pitarangsi C, Amato S, et al. Use of azithromycin for the treatment of Campylobacter enteritis in travelers to Thailand, an area where ciprofloxacin resistance is prevalent. Clinical Infectious Diseases. 1995;21(3):536-41.

(61) Sanders JW, Frenck RW, Putnam SD, Riddle MS, Johnston JR, Ulukan S, et al. Azithromycin and loperamide are comparable to levofloxacin and loperamide for the treatment of traveler's diarrhea in United States military personnel in Turkey. Clinical Infectious Diseases. 2007;45(3):294-301.

(62) Tribble DR, Sanders JW, Pang LW, Mason C, Pitarangsi C, Baqar S, et al. Traveler's diarrhea in Thailand: Randomized, double-blind trial comparing single-dose and 3-day azithromycin-based regimens with a 3-day levofloxacin regimen. Clinical Infectious Diseases. 2007;44(3):338-46.

(63) Steffen R, Sack DA, Riopel L, Jiang ZD, Sturchler M, Ericsson CD, et al. Therapy of travelers' diarrhea with rifaximin on various continents. Am J Gastroenterol. 2003;98(5):1073-8.

(64) Taylor DN, Bourgeois AL, Ericsson CD, Steffen R, Jiang ZD, Halpern J, et al. A randomized, doubleblind, multicenter study of rifaximin compared with placebo and with ciprofloxacin in the treatment of travelers' diarrhea. Am J Trop Med Hyg. 2006;74(6):1060-6. 
(65) DuPont HL, Jiang ZD, Ericsson CD, Adachi JA, Mathewson JJ, DuPont MW, et al. Rifaximin versus ciprofloxacin for the treatment of traveler's diarrhea: A randomized, double-blind clinical trial. Clinical Infectious Diseases. 2001;33(11):1807-15.

(66) Koo D, Maloney K, Tauxe R. Epidemiology of diarrheal disease outbreaks on cruise ships, 1986 through 1993. JAMA. 1996 Feb 21;275(7):545-7.

(67) Mattila L, Siitonen A, Kyronseppa H, Simula II, Peltola H. Risk behavior for travelers' diarrhea among Finnish travelers. J Travel Med. 1995 Jun 1;2(2):77-84.

(68) Hoge CW, Shlim DR, Echeverria P, Rajah R, Herrmann JE, Cross JH. Epidemiology of diarrhea among expatriate residents living in a highly endemic environment. J Am Med Assoc. 1996;275(7):533-8.

(69) Goodyer L. Food and water hygiene for the traveller. Pharmaceutical Journal. 2000;264(7080):134-9.

(70) Curtis V, Cairncross S, Yonli R. Review: Domestic hygiene and diarrhoea-Pinpointing the problem. Tropical Medicine and International Health. 2000;5(1):22-32.

(71) Dupont HL. Traveling internationally: Avoiding and treating travelers' diarrhea. Clinical Gastroenterology and Hepatology. 2010 Jun;8(6):490-3.

(72) DuPont HL. Systematic review: Prevention of travellers' diarrhoea. Aliment Pharmacol Ther. 2008 May;27(9):741-51.

(73) Oldham D, Crawford P, Nichols W, Mott T. What is the best portable method of purifying water to prevent infectious disease? J Fam Pract. 2008;57(1):46-8. 


\title{
Annexe : Foire aux questions sur l'interprétation des résultats de la méthode GRADE
}

\author{
Question : Comment mesure-t-on le niveau de confiance dans l'estimation d'un effet? \\ Réponse : Selon la méthode GRADE, les résultats de l'étude sont regroupés par résultat, puis l'estimation de l'effet est \\ déterminée par méta-analyse. La qualité des données probantes est ensuite évaluée en fonction des cinq critères \\ suivants : \\ - $\quad$ Risque de biais (c.-à-d. limites dans la conception ou la conduite de l'étude); \\ - Imprécision (p. ex. nombre insuffisant de sujets d'étude pour détecter un effet); \\ - Incohérence (c.-à-d. trop grande variabilité entre les résultats de chaque étude); \\ - Caractère indirect (p. ex. importantes différences entre les études dans la manière de mesurer les résultats \\ ou les interventions); \\ - $\quad$ Risque de biais de publication (c.-à-d. les études sans effet ou indiquant des effets non voulus n'ont pas été \\ publiées et ne peuvent donc pas être prises en compte dans l'analyse).
}

Pour chaque critère non satisfait, la qualité est réduite d'un point sur l'échelle à quatre points qui va de « élevée » à « très faible ». De plus, les motifs justifiant chaque abaissement de cote doivent toujours être notés.

Question : Le niveau de confiance à l'égard de l'estimation d'un effet reflète-t-il directement la valeur de la recommandation?

Réponse : Non. La valeur de la recommandation est non seulement fonction de l'estimation de l'effet, mais elle tient également compte de la nature des risques et des avantages, ainsi que des valeurs et des préférences des voyageurs.

\section{Question : Que signifie une recommandation « conditionnelle » dans la pratique?}

Réponse : Dans le présent document, une recommandation fondée sur la méthode GRADE dite « conditionnelle » signifie que le CCMTMV pense que la majorité des voyageurs bien informés choisiraient le plan d'action recommandé, mais qu'une minorité (peut-être une grande minorité) ne le ferait pas. Cela pourrait être dû au fait que l'intervention proposée ne présente que des avantages modestes, que le niveau de confiance à l'égard de l'effet estimé est peu élevé ou que le risque d'effets nocifs potentiels est bien réel. Le profil de résistance aux antimicrobiens est un exemple d'effet nocif potentiel pouvant être associé à la prise d'antibiotiques pour prévenir et traiter la diarrhée du voyageur.

Question : Si l'évaluation selon la méthode GRADE conclut que la confiance à l'égard de l'effet estimé de l'intervention A est élevée, alors que la confiance dans l'estimation de l'effet de l'intervention B est modérée, cela signifie-t-il que l'intervention A est meilleure ou plus efficace que l'intervention $B$ ?

Réponse : Non. Le fait que la qualité des données à l'appui de ces deux interventions soit évaluée séparément signifie, par définition, qu'on ne peut établir de comparaisons directes entre les deux. À titre d'exemple, si l'intervention A est comparée au placebo et que l'intervention B est elle aussi comparée au placebo, on ne peut déduire que l'intervention A est meilleure que l'intervention B, car il s'agit d'une comparaison indirecte.

En revanche, si l'évaluation porte sur des études ayant établi des comparaisons directes entre chaque intervention, il est alors possible d'établir un niveau de préférence. Cependant, cela dépend toujours de l'évaluation globale de l'effet estimé, ainsi que de la qualité des données probantes à l'appui de chaque résultat d'intérêt, sans parler des besoins particuliers de certains groupes tels que les enfants, des valeurs et des préférences des voyageurs, etc. En ce qui concerne la déclaration sur la diarrhée du voyageur, les seules interventions pour le traitement de la diarrhée du voyageur qui ont fait l'objet de comparaisons directes sont les suivantes : lopéramide et antibiotiques comparés aux antibiotiques seuls; azithromycine comparée aux fluoroquinolones et rifaximine comparée aux fluoroquinolones.

Question : Pourquoi certaines données probantes sont-elles évaluées selon la méthode GRADE dans la présente déclaration, alors que d'autres ne le sont pas?

Réponse : Le CCMTMV a conclu que certaines interventions ne se prêtaient pas à une évaluation selon la méthode GRADE, soit à cause d'un manque de solutions plausibles pouvant être utilisées en remplacement de l'intervention à l'étude (p. ex. se laver les mains pour prévenir la diarrhée du voyageur), soit à cause de données probantes insuffisantes (p. ex. quant aux aliments et boissons à privilégier, ou à la prise de probiotiques, pour prévenir la diarrhée du voyageur). Pour ces interventions, le CCMTMV a donc formulé des recommandations en ne s'appuyant que sur la revue de la littérature et sur l'opinion d'experts. 